\title{
Aerodynamic Characteristics of the Combustion Process of Sawdust in a Vortex Furnace with Counter-Swirling Flows
}

\author{
${ }^{1}$ Dzhyoiev R.L., ${ }^{1}$ Redko A.A., ${ }^{2}$ Redko I.A., ${ }^{1}$ Pivnenko Yu.A., ${ }^{1}$ Burda Yu.A., \\ ${ }^{1}$ Gvozdetskii A.V., ${ }^{1}$ Alforov S.A. \\ ${ }^{1}$ Kharkiv National University of Construction and Architecture, Kharkiv, Ukraine \\ ${ }^{2}$ Ukrainian State University of Railway Transport, Kharkiv, Ukraine
}

\begin{abstract}
The aim of this work is to study the working processes of burning the low-quality fuels, namely, the saw dust in the swirling-type furnaces with an opposite twisted motion of the air. The goal was achieved using the physical and mathematical modeling of the flows interaction. The article presented the results of numerical study of aerodynamic characteristics of burning the saw dust in the swirlingtype furnace with the opposite twisted air flows. For the research, the facility was used for the saw dust burning with the air supply into the lower and upper zones of burning. The most essential result of the work was modeling of the working process at the ratio of the flows of the primary air and secondary air without the fuel admixture, equal to 0.2. The tangential rate of the flow changed according to the horizontal sections from 3-5 m/s to $40-42 \mathrm{~m} / \mathrm{s}$ and with respect to the furnace height from $51 \mathrm{~m} / \mathrm{s}$ to 30 $\mathrm{m} / \mathrm{s}$. The average angular rate of the mixture changed relatively the furnace height in the ranges of 171$500 \mathrm{l} / \mathrm{s}$ to $100-300 \mathrm{l} / \mathrm{s}$. The significance of the results obtained consists in determination of the possibility of increasing the efficiency of the work of the furnace facilities at the expense of the introduction of the primary and secondary air flows. In this situation, the optimal ratio of consumptions of primary and secondary air was 0.2 . Thus, in this work the consumption of primary air was $1.285 \mathrm{~kg} / \mathrm{s}$, the consumption of the secondary air was $0.255 \mathrm{~kg} / \mathrm{s}$.
\end{abstract}

Keywords: burning of fuel, swirling-type furnace, particle motion, distribution of pressures in furnace, rate of fuel-air mixture.

DOI: https://doi.org/10.52254/1857-0070.2021.4-52.07

UDC: $62-664.2$

Caracteristicile aerodinamice ale procesului de ardere de rumeguș în cuptorul vortex cu un flux de aer turbionar în contracurent

${ }^{1}$ Gioev R.L., ${ }^{1}$ Redico A.A., ${ }^{2}$ Redico I.A., ${ }^{1}$ Pivnenco Iu.A., ${ }^{1}$ Burda Iu.A.,

${ }^{1}$ Gvozdeții A.V., ${ }^{1}$ Alfiorov S.A.

${ }^{1}$ Universitatea de urbanism și arhitectură din Harkov, Harcov, Ucraina

${ }^{2}$ Universitatea de stat a transportului feroviar din Harcov, Ucraina

Rezumat. Scopul principal al lucrării este de a studia procesele de lucru de ardere a combustibililor de valoare calorică redusă, și anume - rumeguș în cuptoare vortex cu mișcare cu flux de aer turbionar în contracurent. Acest obiectiv a fost atins grație utilizării modelării fizice și matematice a interacțiunii fluxurilor. În articol sunt prezentate rezultatele unui studiu numeric al caracteristicilor aerodinamice ale proceselor de lucru la arderea rumegușului într-un cuptor vortex în contracurent. Pentru cercetare s-a utilizat o instalație pentru arderea rumegușului cu alimentare cu aer în părțile de jos și de sus a zonei de ardere. Cele mai importante realizări ale lucrării sunt rezultatele modelării proceselor de lucru cu un raport de aer primar față de cel secundar fără amestec de combustibil egal cu 0,2. S-a stabilit distribuția reliefului de viteze ale fluxului, traiectoriile mișcării particulelor și distribuția presiunii în volumul camerei de ardere. Au fost studiate fluxurile de particule de până la $=750 \mu \mathrm{m}$ în diametru. S-a stabilit că concentrația de volum a particulelor de-a lungul înălțimii cuptorului variază de la $2,5 \cdot 10^{-}$ ${ }^{3} \mathrm{~kg} / \mathrm{kg}$ la $2,7 \cdot 10^{-3} \mathrm{~kg} / \mathrm{kg}$. Valoarea medie a vitezei absolute a amestecului aer-combustibil este de $28-40 \mathrm{~m} / \mathrm{s}$. Viteza tangențială a fluxului variază de-a lungul secțiunilor orizontale de la $3-5 \mathrm{~m} / \mathrm{s}$ la $40-42 \mathrm{~m} / \mathrm{s}$ și de-a lungul înălțimii cuptorului de la $51 \mathrm{~m} / \mathrm{s}$ la $30 \mathrm{~m} / \mathrm{s}$. Semnificația rezultatelor obținute consta in determinarea posibilității de creștere a eficienței instalațiilor de ardere prin introducerea debitelor de aer primar si secundar. Astfel, raportul optim al debitelor de aer primar și secundar este de 0,2. Deci, în lucrarea dată consumul primar de aer este de 1,285 kg/s și a celui secundar - de $0,255 \mathrm{~kg} / \mathrm{s}$.

Cuvinte-cheie: arderea combustibilului, cuptoare vortex, mișcarea particulelor, distribuția presiunii în cuptor, viteza amestecului combustibil-aer.

( С Джиоев Р.Л., Редько А.А., Редько И.А.,

Пивненко Ю.А., Бурда Ю.А.,

Гвоздецкий А.В., Алфёров С.А., 2021 


\section{Аэродинамические характеристики процесса сжигания опилок в вихревой топке со встречными} закрученными потоками

${ }^{1}$ Джиоев Р.Л., ${ }^{1}$ Редько А.А., ${ }^{2}$ Редько И.А., ${ }^{1}$ Пивненко Ю.А., ${ }^{1}$ Бурда Ю.А., ${ }^{1}$ Гвоздецкий А.В., ${ }^{1}$ Алфёров С.А.

${ }^{1}$ Харьковский национальный университет строительства и архитектуры, Харьков, Украина

${ }^{2}$ Украинский государственный университет железнодорожного транспорта, Харьков, Украина Аннотация. Основной целью работы является изучение рабочих процессов сжигания низкосортных топлив, а именно - древесных опилок, в вихревых топках со встречным закрученным движением воздуха. Поставленная цель была достигнута за счет применения физико-математического моделирования взаимодействия потоков. В статье приведены результаты численного изучения аэродинамических характеристик рабочих процессов сжигания опилок в вихревой топке со встречными закрученными потоками. Для исследований использовалась установка для сжигания древесных опилок с подачей воздуха в нижнюю и верхнюю зону горения. Наиболее важными результатами работы являются результаты моделирования рабочих процессов при соотношении потоков первичного воздуха и вторичного воздуха без примеси топлива, равного 0,2. Установлено распределение полей скоростей потока, траекторий движения частиц и распределение давления в топочном объеме. Изучались потоки с частицами диаметром до $d_{\max }=750$ мкм. Установлено, что объемная концентрация частиц по высоте топки изменяется от $2,5 \cdot 10^{-3}$ кг/кг до $2,7 \cdot 10^{-3}$ кг/кг. Среднее значение абсолютной скорости топливовоздушной смеси составляет 28-40 м/с. Тангенциальная скорость потока изменяется по горизонтальным сечениям от 3 - 5 м/с до 40 - 42 м/с и по высоте топки от 51 м/с до 30 м/с. Средняя угловая скорость смеси изменяется по высоте топки в пределах от 171 - 500 1/с до 100 - 300 1/с. Значимость полученных результатов заключается в определении возможности повышения эффективности работы топочных устройств за счет введения потоков первичного и вторичного воздуха. При этом оптимальное соотношение расходов первичного и вторичного воздуха составляет 0.2. Так, в данной работе расход первичного воздуха составил 1,285 кг/с, расход вторичного воздуха 0,255 кг/с.

Ключевые слова: сжигание топлива, вихревые топки, движение частиц, распределение давлений в топке, скорость топливно-воздушной смеси.

\section{ВВЕДЕНИЕ}

Процессы сжигания древесных отходов исследовались в работах [1-2]. Сжигание древесных отходов осуществляется в слоевых топках и топках с кипящим слоем. Пылевидное топливо сжигается в горелках и вихревых топках. Древесные отходы подразделяются на несколько групп в зависимости от технологии их получения. Опилки относятся к мелким отходам деревообработки, которые прошли сушку до влажности 8-15\%. В зависимости от размеров частиц (от 25 мкм до 500 мкм) опилки разделяют на крупные, мелкие и древесную пыль.

При этом, процесс совместного сжигания биотоплив сопровождается меньшей по сравнению с процессом сжигания угля температурой. Так, температура слоя при совместном сжигании биотоплива составляет от $750{ }^{\circ} \mathrm{C}$ до $800{ }^{\circ} \mathrm{C}$, а при сжигании угля от $900{ }^{\circ} \mathrm{C}$ до $950{ }^{\circ} \mathrm{C}$. К тому же, значительную роль в процессах сжигания твердых топлив играет способ подачи первичного воздуха. [313].

В [14] изучены гидродинамические режимы и характеристики конического закрученного псевдоожиженого слоя и произведено сравнение влияния тангенциальной и осевой скорости на слой. Также исследован эффект различной высоты слоя и предложена математическая модель прогнозирования минимальной скорости псевдоожижения ( $\left.U_{m f}\right)$ и перепада давления в слое $\left(\Delta p_{b}\right)$. При этом установлено, что оба способа подачи воздуха (осевой и тангенциальный) оказывают влияние на гидродинамику. В работе [15] для сжигания биомассы применен осевой способ подачи воздуха с кольцевым распределителем.

Кроме способа подачи воздуха на процесс сжигания биотоплива влияют размер его частиц и высота слоя. Исследования [3] показывают, что частицы размером 425-600 мкм перемещаются по всей высоте топки, в то время как частицы размером 600-850 мкм поднимаются лишь на небольшую высоту (25 мм). При этом для частиц в 850-1180 мкм наблюдается процесс барботажа по всей высоте топочного пространства. Установлено, что частицы размером 425-600 мкм в толщине слоя 40 мм являются самым эффективным способом сжигания биотоплив в вихревых топках.

В работе [15] приведены результаты исследований в области соотношения 
расходов потоков превышающих значения 0.5. В этом случае реализуется устойчивая квазицилиндрическая макроструктура потоков: наружного нисходящего и внутреннего восходящего. Кривая распределения тангенциальной составляющей скорости близка по форме к вихрю Ренкина. Осевая составляющая скорости по всей высоте аппарата в приосевой области направлена вверх и характеризуется максимальным значением. При сжигании топлива в вихревой топке со встречными закрученными потоками соотношение расходов потоков определяется процессами горения и значение его меньше чем 0.5. Данные о составляющих скорости и структуре потоков в литературе не приведены. В отличии от [15] данная работа рассматривает аэродинамические процессы в топке с первичным и вторичным воздухом в соотношении 0.2 .

Целью настоящей работы является изучение аэродинамических характеристик процесса сжигания низкосортных топлив, а именно - древесных опилок, в вихревых топках со встречным закрученным движением воздуха при соотношении расходов потоков воздуха 0.2 .

\section{I. МЕТОДЫ ИССЛЕДОВАНИЯ}

Основой методики исследований является физико-математические методы теоретических исследований процессов сжигания низкосортных топлив на основе законов и уравнений физико-химической кинетики, аэродинамики и тепломассообмена, а именно, физико-математическое моделирование взаимодействия струй. Лабораторные и натурные исследования базируются на современных теориях постановки, исполнения, обработки и получения данных результатов теплофизического эксперимента.

Расчеты выполнялись с помощью системы дифференциальных уравнений в частных производных, подготовленную с усредненных за Рейнольдсом уравнений Навье-Стокса, двух уравнений дифференциальной модели турбулентности $k-\varepsilon$ типа, уравнений сохранения для безразмерных функций Шваба-Зельдовича и для пульсаций этих функций и интегро-дифференциального уравнения переноса излучения.

$$
\begin{gathered}
\rho \frac{\partial u_{j}}{\partial x_{j}}=S_{n}, \\
\rho \frac{\partial u_{j} u_{j}}{\partial x_{j}}-\frac{\partial p}{\partial x_{i}}-\frac{\partial \tau_{i j}}{\partial x_{j}}=S_{f i}, j=1,2,3,(2) \\
\rho \frac{\partial u_{j} h}{\partial x_{j}}-\frac{\partial}{\partial x_{j}}\left(\frac{\mu}{P r}+\frac{\mu_{m}}{P r_{m}}\right) \frac{\partial h}{\partial x_{j}}=S_{q}, j=1,2,3(3) \\
\rho \frac{\partial u_{j} k}{\partial x_{j}}-\frac{\partial}{\partial x_{j}}\left(\mu+\frac{\mu_{m}}{\sigma_{k}}\right) \frac{\partial k}{\partial x_{j}}-\rho(G-\varepsilon)=0, \\
j=1,2,3 \\
\rho \frac{\partial u_{j} \varepsilon}{\partial x_{j}}-\frac{\partial}{\partial x_{j}}\left(\mu+\frac{\mu_{m}}{\sigma_{\varepsilon}}\right) \frac{\partial \varepsilon}{\partial x_{j}}-\rho\left(C_{\varepsilon 1} G-C_{\varepsilon 2} \varepsilon\right) \frac{\varepsilon}{k}=0 \\
j=1,2,3 \\
\frac{\partial\left(\vec{u} f_{n}\right)}{\partial x_{j}}=\frac{\partial}{\partial x_{j}}\left(\frac{\mu_{m}}{\sigma_{m}} \frac{\partial f_{n}}{\partial x_{j}}\right)+S_{n}, j=1,2, n=1,2,3(6) \\
\rho \frac{\partial\left(\vec{u} g_{n}\right)}{\partial x_{j}}=\frac{\partial}{\partial x_{j}}\left(\frac{\mu_{m}}{\sigma_{m}} \frac{\partial g_{n}}{\partial x_{j}}\right)+C_{g} \mu_{m}\left(\frac{\partial f_{n}}{\partial x_{j}}\right)^{2}-C_{d} \rho \frac{\varepsilon}{k} g_{n} \\
j=1,2, n=1,2,3
\end{gathered}
$$

где $\mu_{j}$ - декартовые компоненты вектора усредненной скорости газа;

$x_{j}$ - декартовые координаты;

$S_{n}$ - источник массы, соответствующий переносу массы в газовую фазу от реагирующих частиц;

$\rho$ - плотность газа;

$p$ - давление газа;

$\tau_{i j}$ - компоненты тензора напряжений;

$$
\tau_{i j}=\left(\mu+\mu_{m}\right)\left(\frac{\partial u_{i}}{\partial x_{j}}+\frac{\partial u_{j}}{\partial x_{i}}\right)
$$

$\mu$ - динамическая вязкость;

$\mu_{m}$ - турбулентная вязкость, определяется по формуле Колмогорова-Прандтля; 


$$
\mu_{m}=C_{\mu} \rho \frac{k^{2}}{\varepsilon}
$$

$C_{\mu}$ - эмпирический коэффициент;

$k$ - кинетическая энергия турбулентности;

$$
k=\frac{1}{2} u_{i}^{\prime} u_{i}^{\prime} ;
$$

$\boldsymbol{u}_{i}^{\prime}$ - возмущение скорости газа относительно усредненного значения;

$\varepsilon$ - удельная скорость диссипации кинетической энергии турбулентности;

$$
\varepsilon=\frac{1}{2} v\left(\frac{\partial u_{j}^{\prime}}{\partial x_{i}}+\frac{\partial u_{i}^{\prime}}{\partial x_{j}}\right)^{2}
$$

$v$ - коэффициент кинематической вязкости; $S_{f j}$ - источник количества движения, обусловленный межфазным взаимодействием; $h$ - удельная энтальпия;

$$
h=\sum_{i} Y_{i}\left(h_{f i}^{0}+\int_{T^{0}}^{T} c_{p i}(T) d T\right)
$$

$Y_{i}$ - массовая часть химического компонента $i$; $h_{f i}^{0} \quad-$ удельная энтальпия осветления химического компонента $i$;

$T$ - температура газа;

$T^{0}$ - стандартная температура;

$c_{p i}$ - удельная теплоемкость химического компонента $i$ при постоянном давлении;

$\operatorname{Pr}$ - число Прандтля;

$P r_{T}$ - турбулентное число Прандтля;

$S_{q}$ - источник теплоты, обусловленный межфазным взаимодействием с частицами; $\sigma_{k}, \sigma_{\varepsilon}, C_{\varepsilon 1}, C_{\varepsilon 2}$ - эмпирические коэффициенты;

$G$ - член, характеризующий генерацию кинетической энергии турбулентности;

$$
G=\mu_{T} \frac{\partial u_{i}}{\partial x_{j}}\left(\frac{\partial u_{i}}{\partial x_{j}}+\frac{\partial u_{j}}{\partial x_{i}}\right)
$$

$\sigma_{T}, C_{g}, C_{d}$ - эмпирические коэффициенты;

$\beta_{0}=k_{a}+\sigma_{s}-$ объемный коэффициент угасания;

$k_{a}$ - объемный коэффициент поглощения;

$\sigma_{s}$ - объемный коэффициент рассеивания;

I - интенсивность излучения; $\vec{r}$ - радиус-вектор произвольной луча в угловом направлении распространения излучения $\vec{s}$;

$\omega_{0}=\sigma_{s} / \beta_{0}-$ альбедо рассеивания;

$I_{b}=\sigma T^{4} / \pi-$ интенсивность излучения абсолютно черного тела;

$\sigma$ - постоянная Стефана-Больцмана;

$\Omega^{\prime}$ - единичный вектор телесного угла, который характеризует направление распространения теплового излучения из-за рассеивания фотонов $\vec{s}$;

Безразмерная функция Шваба - Зельдовича определяется выражением:

$$
\begin{gathered}
f_{n}=\frac{Z_{q}-Z_{q . o}}{Z_{q . F_{1}}-Z_{q . o}} ; \\
f_{1}+f_{2}+f_{0}=1 ;
\end{gathered}
$$

где $Z_{q}$ - массовая частица элемента $q$; индексы « $F_{I} »$ и «O» относятся к первичному топливу и окислителя соответственно.

Все термохимические скаляры $\varphi_{i}$ (массовые части химических компонентов, плотность и температура) зависят исключительно от $f_{n}$ :

$$
\phi_{i}=\int_{0}^{1} \int_{0}^{1} \phi_{i}\left(f_{1}, f_{2}, h\right) P_{1}\left(f_{1}\right) P_{2}\left(f_{2}\right) d f_{1} d f_{2}
$$

где $h$ - мгновенная энтальпия;

$P_{n}\left(f_{n}\right)$ - функция ПРВ, описывающая вероятность того, что значение переменной $\varphi_{i}$ находится между $\varphi$ и $\varphi+\Delta \varphi$, и аппроксимирует $\beta$-функцию.

Уравнение состояния смеси идеальных газов:

$$
p=R \rho T \sum_{i} \frac{Y_{i}}{M_{i}}
$$

где $R$ - универсальная газовая постоянная; $M_{i}$ - молекулярная масса химического компонента $i$.

Массовую долю частиц по фракциям (до 750 мкм) и количество частиц в процентном соотношении определяли аналогично [17] с применением распределения РозинаРаммлера:

$$
Y_{d}=e^{-(d / \bar{d})^{n}},
$$


где $Y_{d}-$ массовая часть частиц с диаметром d;

$\bar{d}$ - медианный диаметр частиц;

$n$ - параметр разделения.

Траектории частиц моделировались путем интегрирования уравнения баланса сил, действующих на частицу, которое уравнивает инерцию частицы с силой аэродинамического сопротивления

$\frac{d u_{p j}}{d t}=-\frac{3 \rho C_{R}}{4 \rho_{p} d_{p}}\left(u_{p j}-u_{j}\right) \sqrt{\sum_{j}\left(u_{p j}-u_{j}\right)^{2}}, j=1,2,3,(20)$

где $u_{p j}$ - декартовые компоненты вектора скорости частицы;

$t$ - время;

$\rho$ - плотность газа;

$C_{R}-$ коэффициент аэродинамического сопротивления частицы;

$\boldsymbol{R} \boldsymbol{e}_{p}$ - относительное число Рейнольдса частицы;

$\rho_{p}-$ плотность частицы;

$d_{p}$ - диаметр частицы;

$u_{j}$ - декартовые компоненты вектора скорости газа [17].

При этом тепломассообмен частиц описывался тремя моделями: моделью теплообмена инертной части; моделью выхода летучих и моделью выгорания коксового остатка.

Модель теплообмена инертной части применялась до тех пор, пока температура частицы $T_{p}$ не превышала температуру выхода летучих $T_{V}$. Температура частицы полагалась постоянной по объему и изменялась в соответствии с балансом тепла, определяются уравнением:

$$
m_{p} c_{p} \frac{d T_{p}}{d t}=\alpha S_{p}\left(T_{\infty}-T_{p}\right)
$$

где $m_{p}$ - масса частицы;

$c_{p}$ - удельная теплоемкость частицы;

$T_{p}$ - температура частицы;

$t$ - время;

$\alpha$ - коэффициент теплоотдачи;

$S_{p}$ - площадь поверхности частицы;

$T_{\infty}$ - локальная температура газа.

Модель выхода летучих использовалась, когда температура частицы достигала температуры начала выхода летучих $\boldsymbol{T}_{v}$ и действовала до тех пор, пока масса частицы $m_{p}$ превышала начальную массу нелетучих компонентов в частице.

$$
T_{p} \geq T_{v} \text { и } m_{p}>\left(1-f_{v, 0}\right) m_{p, 0},
$$

где $f_{v, 0}-$ массовая доля летучих, изначально присутствующая в частице.

Тепломассообмен частицы во время выхода летучих описывался системой уравнений

$$
\begin{aligned}
m_{p} c_{p} \frac{d T_{p}}{d t} & =\alpha S_{p}\left(T_{\infty}-T_{p}\right)+\frac{d m_{p}}{d t} h_{v}, \\
\frac{d m_{p}}{d t} & =-f_{v, 0} m_{p, 0} k,
\end{aligned}
$$

где $h_{v}$ - удельная теплота осветления летучих;

$m_{p, 0}-$ начальная масса частицы;

$k$ - кинетическая скорость реакции горения, что определяется по уравнению Аррениуса

$$
k=A_{1} e^{-\left(E / R T_{p}\right)},
$$

где $A_{1}$ - предэкспотенциальный множитель; $e$ - энергия активации;

$R$ - универсальная газовая постоянная.

Данная модель выхода летучих предусматривает, что скорость выхода летучих первоначально зависит от количества летучих, оставшегося в частице.

Модель выгорания частиц использовалась после окончания выхода летучих и до тех пор, пока не выгорит весь их остаток:

$$
\left(1-f_{v, 0}-f_{\kappa}\right) m_{p, 0}<m_{p}<\left(1-f_{v, 0}\right) m_{p, 0}
$$

где $f_{\kappa}-$ массовая доля кокса, изначально присутствующего в частице.

Данная модель выгорания предусматривает, что скорость поверхностной реакции определяется как кинетической, так и диффузной составляющими.

Тепломассообмен частицы при выгорании коксового остатка описывался системой уравнений 


$$
\begin{gathered}
m_{p} c_{p} \frac{d T_{p}}{d t}=\alpha S_{p}\left(T_{\infty}-T_{p}\right)-f_{h} \frac{d m_{p}}{d t} H_{u}, \\
\frac{d m_{p}}{d t}=-S_{p} p_{O_{2}} \frac{D_{0} R}{D_{0}+R},
\end{gathered}
$$

где $f_{h}$ - эмпирические коэффициент;

$H_{u}$ - удельная теплота сгорания кокса;

$S_{p}$ - площадь поверхности частицы;

$p_{O_{2}}-$ парциальное давление кислорода в газе;

$D_{o}-$ коэффициент диффузионной скорости реакции горения определяется выражением:

$$
D_{0}=C_{1} \frac{\left[\left(T_{p}+T_{\infty}\right) / 2\right]^{0,75}}{d_{p}},
$$

где $C_{1}-$ эмпирический коэффициент;

$d_{p}$ - диаметр частиц;

$R$ - коэффициент кинематической скорости реакции горения определяется выражением:

$$
R=C_{2} e^{-\left(E / R T_{p}\right)}
$$

где $C_{2}$ - предэкспоненциальный множитель.

Подробное описание методики расчета приведено в [17].

\section{II. РЕЗУЛЬТАТЫ ИССЛЕДОВАНИЙ}

Численно исследовались процессы сжигания древесных опилок диаметром от $d_{\min }=25$ мкм до $d_{\max }=750$ мкм в вихревой топке теплопроизводительностью 2,6 MBT (рис 1).

Принцип работы топки следующий: топливно-воздушная смесь подается в пространство топки через патрубок 2 в нижней части топки. При этом смесь направляется вертикально вверх. В то же время вторичный воздух подается через патрубок 1 , располагающийся в верхней части топки. Шлак удаляется из топочного пространства через патрубок 3. Зола удаляется из расширителя, находящегося в верхней части топки.

Указаны сечения по высоте топки с шагом 1 м, в которых определялись значения скоростей топливно-воздушной смеси (рис 1).

Граничные условия следующие: на входящих участках - значения независимых переменных; «мягкие» граничные условия. При этом задавались начальные условия для каждой частицы: ее положение (координаты), скорость, диаметр, температура, массовый их расход.

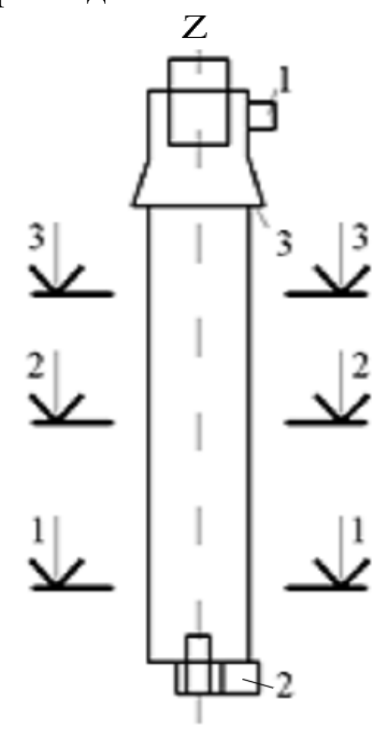

a

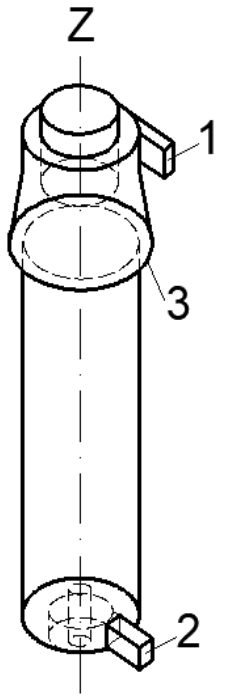

6

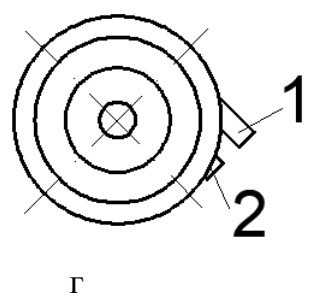

а - вид сбоку; б - изометрия; в - вид сверху. ${ }^{1}$

(1 - патрубок подачи вторичного воздуха;

2 - патрубок подачи топлива и воздуха; 3 - щель для шлака)

Рис. 1. Принципиальная сетка установки.

В качестве топлива применялись древесные опилки.

Элементарный состав топлива следующий: $\mathrm{C}=50 \% ; \mathrm{H}=6 \% ; \mathrm{B}=43,5 \% ; \mathrm{N}=0,5 \%$. Технический состав: влажность 10\%; зольность на сухую массу Ас $=0,6 \%$, на рабочую $\mathrm{Ap}=0,54 \%$; выход летучих на горючую массу 85\%, на рабочую $\mathrm{Vp}=$ $76,041 \%$

Характеристики топлива следующие: высшая теплота сгорания (на рабочую массу) 17,676 МДж/кг; теплоемкость 1,70 кДж/кг;

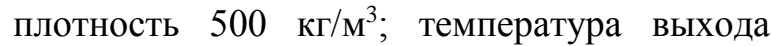
летучих $200{ }^{\circ} \mathrm{C}$. Средний диаметр опилок $\left(d_{50}\right)$ составил 1,5 мм, коэффициент полидисперсности 0,12. Расход опилок составил 0,145 кг/c, расход первичного воздуха, подающегося снизу топки составляла 1,285 кг/с, расход вторичного воздуха 0,255 кг/с.

1.Appendix 
Результаты расчета показывают, что степень выгорания легких опилок составляет $100 \%$. При этом степень выгорания кокса в частицах, которые выносятся, также составляет $100 \%$.

Вынос частиц составляет $56 \%$, улавливание частиц - $31 \%$. Около $13 \%$ частиц накапливается в топочном объеме длительное время до полного выгорания. Выход кислорода составляет 9,5\%, температура уходящих газов $1473{ }^{\circ} \mathrm{C}$. Гидравлическое сопротивление при движении частиц внутри установки составляет около 2,5 кПа.

Объемная концентрация твердой фазы внизу топки:

$$
\beta=\frac{1}{Q_{T}} \cdot \frac{d p}{d u}=2 \cdot 10^{-2}
$$

Плотность дисперсности потока:

$$
\rho_{I}=\frac{1}{g} \cdot \frac{\Delta p}{\Delta y}=10 \kappa \Gamma / \mathrm{M}^{3}
$$

Расходная концентрация дисперсного материала:

$$
\mu=\frac{M_{T}}{M_{B}}=0,112 \kappa \Gamma / \kappa \Gamma
$$

Расход топлива в топке

$$
m_{T}=\frac{M_{T}}{F}=0,514 \kappa \Gamma / \mathrm{M}^{2}
$$

На рис. 2А и рис. 2.Б указаны траектории частиц диаметром 25 мкм и 750 мкм с различным массовым содержанием кокса.

Массовое содержание отображается в процентном соотношении согласно шкалы, приведенной на рисунках и окрашено в различные цвета.

В зависимости от диаметра частиц сгорание кокса происходит в различных местах топочного объема.

Как видно, сжигание более крупных частиц происходит в верху топки, а именно - вблизи выхода из нее.

Частицы диаметром 25 мкм сгорают вблизи входа в топку в нижней ее части.
В верхней части топки увеличивается количество частиц, которые длительное время витают в топочном объеме.

Шn мm 2 mимедены результаты расчетов

${ }^{2}$ Appendix 1 давления газов в топочном объеме. При этом процентное содержание кокса в составе частиц отображено цветовой шкалой. Давление газов изменяется незначительно как по высоте, так и по сечению топки.

Графическое отображение результатов расчетов параметров топливно-воздушной смеси получено при помощи программы конечно-элементного анализа ANSYS.
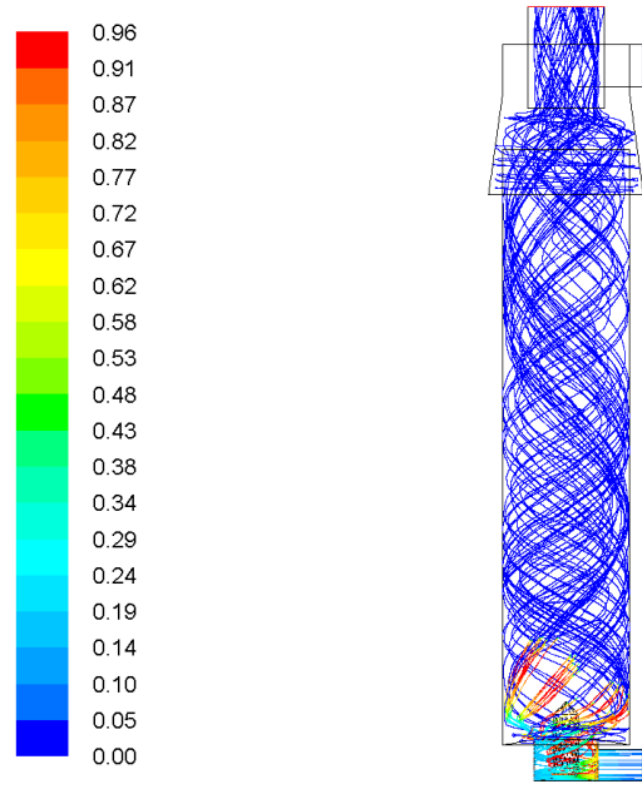

Рис. 2.А. Траектории частиц с начальным диаметром 25 мкм. ${ }^{2}$

На рис. 4 представлено распределение тангенциальной составляющей скорости в отопочном объеме. Значение скорости окрашено в различные цвета согласно цветовой шкале.

Значения тангенциальной составляющей скорости в большей части объема топки изменяется от 30 до $45 \mathrm{~m} / \mathrm{c}$.

При этом наблюдается высокие значения тангенциальной скорости в подводящем патрубке (до $250 \mathrm{~m} / \mathrm{c}$ ), в то время, как около оси тангенциальная скорость крайне низкая и может достигать отрицательных значений, что свидетельствует об обратном направлении движения воздушного потока.

На оси топки и вблизи ее стенок скорость ниже, чем на расстоянии 0,25 - 0,3 диаметра топки. 


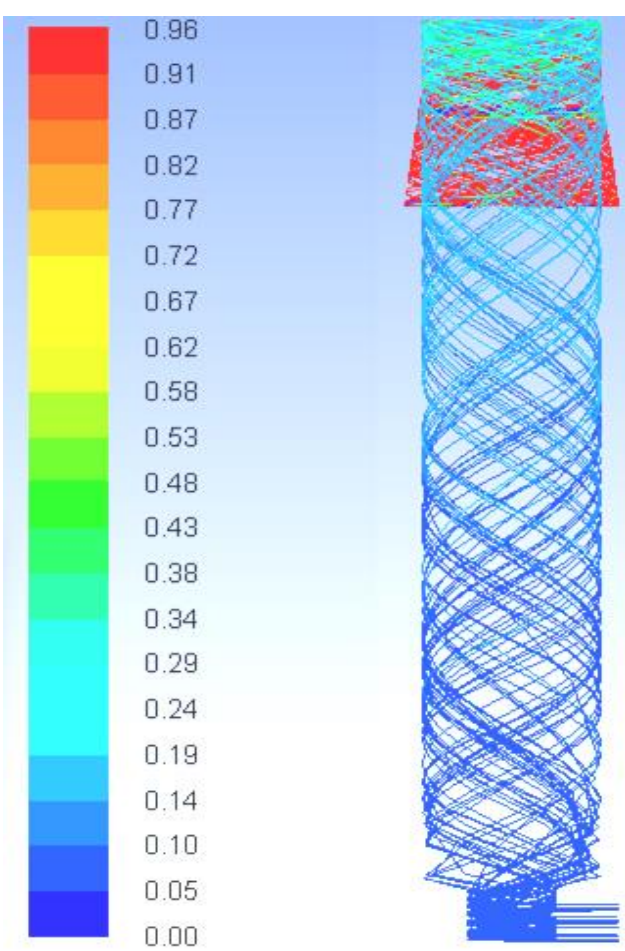

Рис. 2.Б. Траектории частиц с начальным диаметром 750 мкм. $^{3}$
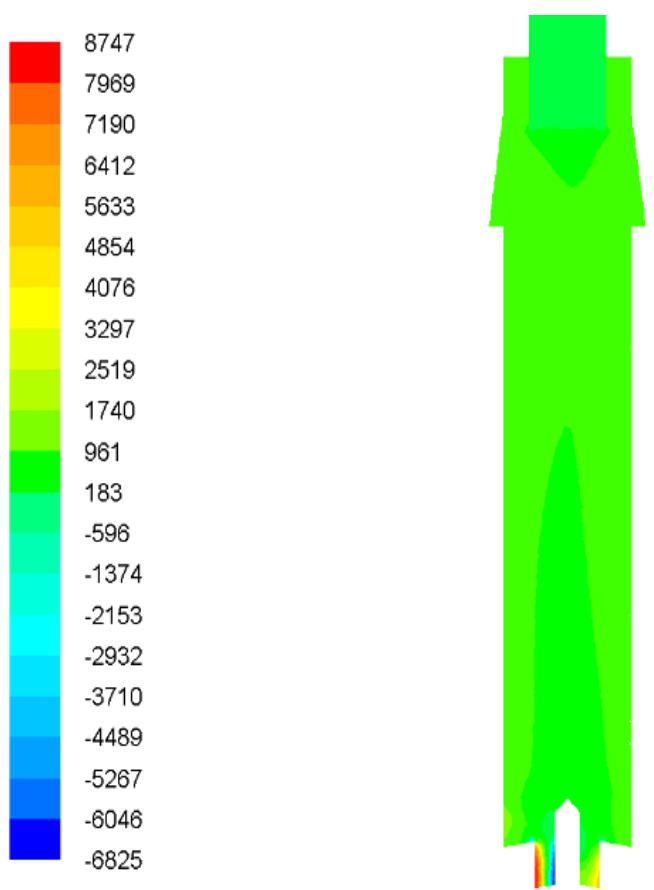

Рис. 3. Распределение избыточного статического давления (Па) в продольном сечении топки. ${ }^{4}$

3,4,5,6,Appendix 1 ,ость определялась по формуле:

$$
\omega=\frac{V_{\delta_{V}}}{R} 1 / \mathrm{c}
$$
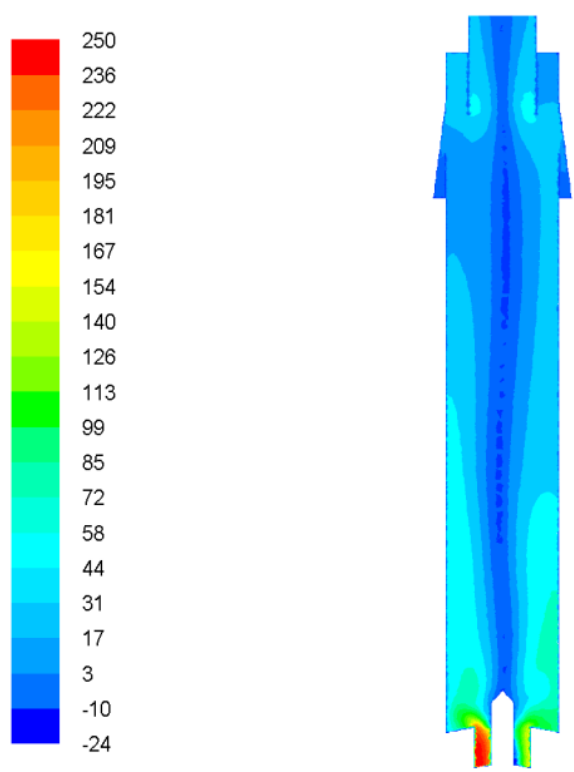

Рис. 4. Распределения тангенциальной составляющей скорости (м/с) в топочном объеме. 5

Распределение осевой и тангенциальной компонент скорости топливо-воздушного потока в сечении 2 топки указано на рис. 5 и рис. 6. Цветовая шкала отображает значение скоростей потока в данном сечении. При этом тангенциальная скорость $V_{\delta_{\mathrm{V}}}$ достигает значений 30 - 45 м/с. На оси топки значения $V_{\delta_{v}}$ составляют 5 - $10 \mathrm{M} / \mathrm{c}$. Вблизи стенки скорость $V_{\delta_{v}}$ также понижается до 5 - $10 \mathrm{M} / \mathrm{c}$. В целом по сечению $V_{\delta_{v}}$ изменяется от $5 \mathrm{~m} / \mathrm{c}$ до $45 \mathrm{M} / \mathrm{c}$. Средняя скорость $V_{\delta_{v}}$ по сечениям следующая: в сечении $1-51 \mathrm{M} / \mathrm{c}$; в сечении 2 39 м/с; в сечении $3-30$ м/с.

Угловая скорость изменяется по сечению 2 топки от 100 - 200 1/с до 225 - 300 1/c.

По высоте топки угловая скорость изменяется незначительно по сечению 3 от 150 - $3001 / \mathrm{c}$.

При этом средняя угловая скорость изменяется по высоте топки в пределах от 171 - 500 1/с до 100 - 300 1/c.

По сечению:

$$
\omega_{\min }=\frac{10 \mu / c}{0,1 \mu}=1001 / \mathrm{c}
$$




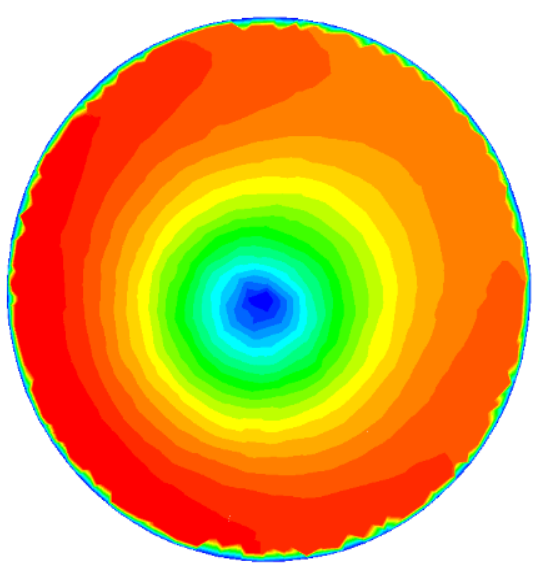

Рис. 5. Распределения тангенциальной составляющей скорости $(\mathrm{m} / \mathrm{c})$ в сечении 2 топки. ${ }^{6}$

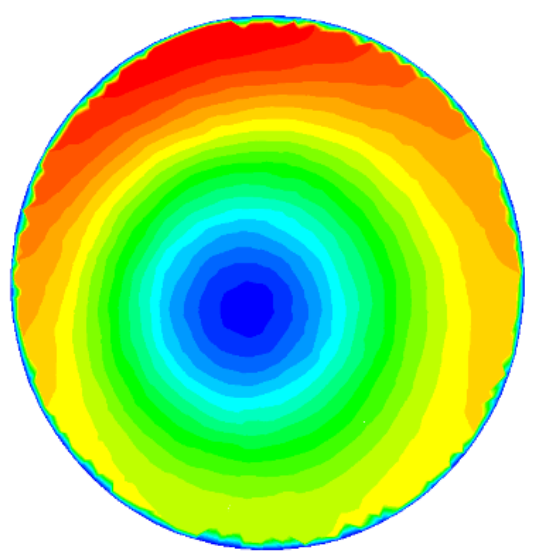

Рис. 6. Распределения осевой компоненты скорости (м/с) в сечении 2 топки. ${ }^{7}$

$$
\text { 7.Appendix } 1 \omega_{\max }=\frac{45 \mu / c}{0,2 M}=2251 / \mathrm{c} .
$$

Изменение расхода газа возможно в диапазоне от 15 до 50\%, изменение концентрации пыли от 20 до $200 \quad$ г/ $\mathrm{m}^{3}$. Запыленность воздуха изменялась от 17 г/м ${ }^{3}$ до $35 \Gamma / \mathrm{M}^{3}[18$ - 24].

В пылеуловителе диаметром 200 мм при изменении объемного расхода от $502 \mathrm{~m}^{3} /$ час до $1041 \mathrm{~m}^{3} /$ час гидравлическое сопротивление составляет от 1,15 кПа до 2,55 кПа, при

средней скорости воздуха в сечении 4,4 - 9,2 м/с при угловой скорости у стенки $192-294$ 1/c. При этом установлено распределение угловой скорости движения воздуха по сечению аппарата [24]. Концентрация пыли составляет 17-35 $\Gamma / \mathrm{M}^{3}, \quad$ эффективность сепарации составляет 98-99\%.

Среднее значение осевой скорости по высоте топки изменяется так: $31 \mathrm{~m} / \mathrm{c}$ в сечении $1 ; 29 \mathrm{м} / \mathrm{c}$ в сечении $2 ; 28 \mathrm{м} / \mathrm{c}$ в сечении 3.

По сечению 2 топки осевая скорость изменяется от 5 - 10 м/с до $30-40$ м/с вблизи стенки. На выходе из топки абсолютная скорость составляет $70-90 \mathrm{~m} / \mathrm{c}$.

\section{III. ЗАКЛЮЧЕНИЕ}

Результаты численного исследования, полученные в настоящей работе позволяют модернизировать существующие топки за счет ввода в них потоков первичного и вторичного воздуха с соотношением расходов данных воздушных потоков 0.2 .

Аэродинамические характеристики топливо-воздушной смеси при иных соотношениях первичного и вторичного воздуха приведены в [15]. При этом распределение скоростей потоков в топочном объеме рассмотрено впервые.

Данной работой становлена зависимость содержания кокса от размеров частиц и разпределение их скоростей по высоте вихревой топки. Так, наивысшее содержание кокса в частицах с начальным диаметром 25 мкм наблюдается в нижней части топки, в то время как в частицах с начальным размером 750 мкм - в ее верхней части. При этом, средние значения тангенциальной составляющей скорости составило от 30 до 51 м/с, осевой скорости $-28-31 \mathrm{~m} / \mathrm{c}$. Угловая скорость по высоте топки изменялась в пределах от 100 до $500 \mathrm{l} / \mathrm{c}$. Значение тангенциальной составляющей монотонно возрастает от при осевой области к стенке. Осевая составляющая также возрастает в при осевой области по всей высоте топки.

\section{APPENDIX 1 (ПРИЛОЖЕНИЕ 1)}

${ }^{1}$ Fig. 1. Schematic diagram of the installation: a - side view; b - isometry; c - top view.

${ }^{2}$ Fig. 2.A. Trajectories of particles with an initial diameter of $25 \mu \mathrm{m}$, colored according to the mass fraction of coke in their composition.

${ }^{3}$ Fig. 2.B. Trajectories of particles with an initial diameter of $750 \mu \mathrm{m}$, colored according to the mass fraction of coke in their composition. 
${ }^{4}$ Fig. 3. Distribution of excess static pressure $(\mathrm{Pa})$ in the longitudinal section of the furnace.

${ }^{5}$ Fig. 4. Distribution of the tangential velocity component $(\mathrm{m} / \mathrm{s})$ in the furnace volume.

${ }^{6}$ Fig. 5. Distribution of tangential velocity $(\mathrm{m} / \mathrm{s})$ in section 2 of the furnace.

${ }^{7}$ Fig. 6. Distribution of the axial velocity component $(\mathrm{m} / \mathrm{s})$ in section 2 of the furnace.

\section{Литература (References)}

[1] Geletukha. G.G., Zheleznaya T.A. Obzor tekhnologii gazifikatsii biomassy, [Biomass Gasification Technology Review]. Ekotekhnologii I resursosberezhenie Ecotechnology and resource conservation, 1998, no.2, pp. 27-29. (In Russian).

[2] Pivnenko Y.O. Pidvyshchennya efektyvnosti spalyuvannya derevnykh vidkhodiv u topkakh kyplyachogo sharu opalyuval'nykh kotel'nykh. Diss. kand. tekh. nauk [Improving the efficiency of burning wood waste in fluidized bed furnaces of boiler houses. Ph.D. in Technology diss.]. Kharkov, 2018. 164 p.

[3] Belousov A.S., Sagin B.S., Lopakov A.V., Markov R.A., Sagin V.B. Experimentalnoe issledovanie poley skorostey $v$ apparate so vstrechnymi zakrychennymi potokami. [Experimental study of velocity fields in a vehicle with counter-swirling flows.], Advances in chemistry and chemical technology, 2012, no. 26, p. 131 (In Russian).

[4] Sun P., Hui S., Gao Z., Zhou Q., Tan H., Zhao Q., $\mathrm{Xu}$ T. Experimental investigation on the combustion and heat transfer characteristics of wide size biomass co-firing in $0.2 \mathrm{MW}$ circulating fluidized bed. Applied Thermal Engineering. 2013, vol. 2, no.52. pp. 284-292.

[5] Basu P. Circulating Fluidized Bed Boilers: Design, Operation and Maintenance. Springer International Publishing. vol. 6. no.2. 2015 pp.58-64.

[6] Blaszczuk A., Nowak W., Krzywanski J. Effect of bed particle size on heat transfer between fluidized bed of group b particles and vertical rifled tubes. Powder Technol. 2017, vol.11, no.316, pp. 111-122. doi: 10.1016/j.powtec.2016.12.027.

[7] Madejski P., Taler D., Taler J. Numerical model of a steam superheater with a complex shape of the tube cross section using Control Volume based Finite Element Method. Energy Convers. Manag. 2016, vol. 13, no.118, pp. 179-192. doi: 10.1016/j.enconman.2016.03.069

[8] Brems A., Cáceres G., Dewil R., Baeyens J., Pitié F. Heat transfer to the riser-wall of a circulating fluidised bed (CFB) Energy. 2013, vol. 7, no.50, pp. 493-500. doi: 10.1016/j.energy.2012.10.037.

[9] Win K.K., Nowak W., Matsuda H., Hasatani M., Bis Z., Krzywanski J., Gajewski W. Transport Velocity of Coarse Particles in Multi-Solid
Fluidized Bed. J. Chem. Eng. Jpn. 1995, vol. 5, no.28, pp.535-540. doi: 10.1252/jcej.28.535.

[10] Muskała W., Krzywański J., Sekret R., Nowak W. Model research of coal combustion in circulating fluidized bed boilers. Chem. Process Eng. 2008, vol. 19 , no. 29, pp. 473-492.

[11] Oka, S. Fluidized Bed Combustion,, Inc., New York. 2003, vol. 3, pp. 105-107.

[12] Al-Abbas A.H., Naser J., Dodds D. CFD modelling of air-fired and oxy-fuel combustion in a large-scale furnace at Loy Yang A brown coal power station. Fuel. 2012, vol. 10, no. 102, pp. 646-665.

[13] Hwang M., Kim S., Kim G., Lee B., Song J., Park M., Jeon C. Simulation studies on direct ash recycling and reburning technology in a tangentially fired $500 \mathrm{MW}$ pulverized coal boiler. Fuel. 2013, vol. 7, no. 114. pp. 78-87.

[14] Anufriev I., Salomatov V., Anikin Y., Krasinsky D., Sharypov O., Enkhjargal Kh. Modeling of Aerodynamics in Vortex Furnace. Cleaner Combustion and Sustainable Word. Proceedings of the 7th International Symposium on Coal Combustion. Tsinghua University Press, 2011, vol, 910 pp. 687-693.

[15] Kaewklum, R., Kuprianov, V.I. and Douglas, P.L. Hydrodynamics of air-sand flow in a conical swirling fluidized bed: A comparative study between tangential and axial air entries, Energy Conversion and Management, 2009, vol. 12, no. 50, pp. 2999-3006.

[16] Silin V.E., Ryzhkov A.F. Osobennosti nizkotemperaturnogo goreniya drevesnogo topliva $v$ sovremennykh energoustanovkakh. [Features of low-temperature combustion of wood fuel in modern power plants.], Prom.energetica - Prom. energetics, 2008, no. 10, p. 117 (In Russian).

[17] Redko A.A., Redko I.A., Redko A.F. Szhiganie tverdogo topliva v vikhreoi topke so vstrechnymi zakruchennymi potokami [Combustion of solid fuel in a vortex furnace with countering swirled flows], Problemy regional'noi energetiki Regional energy problems, 2017, no. 35, p. 33-44 (In Russian).

[18] Badzioch S., Hawksley P. G. W. Kinetics of Thermal Decomposition of Pulverized Coal Particles. Process Design and Development, 1970, vol. 7, pp. 521-530.

[19] Lazarev V.A. Cyclones and vortex dust collectors: a handbook. N. Novgorod, 2006. 320 p.

[20] Sazhin B.S., Gydim L.I., Vortex dust collectors. Moscow, Khimiya, 1995. 144 p.

[21] Mohideen, M.F., Sreenivasan, B., Sulaiman, S.A., Raghavan, V.R. Heat transfer in a swirling fluidized bed with Geldart type-D particles, Korean J. Chem. 2012, vol. 7, no 29. pp. 862-867.

[22] Mohideen M. F., Faiz, M., Salleh H., Zakaria H., Raghavan, V. R. Drying of oil palm frond via 
swirling fluidization technique. In Proceedings of the World Congress on Engineering, 2011, vol. 3, no 18. pp. 2375-2380.

[23] Zakaria, J. H., Zaid M., Hashemi M. H., Mohideen Batcha M. F., Asmuin, N. Drying of sponge media using swirling fluidized bed dryer. Applied Mechanics and Materials, 2014, vol. 4, pp. 644- 648.

\section{Сведения об авторах.}

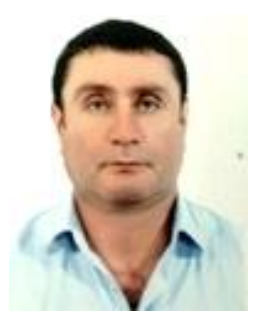

Джиоев

Рафаэль Леванович

аспирант каф. ТГВ и ТВЕР, ХНУСА. Научные интересы: низкопотенциальная

энергетика, солнечная энергия, теплофизика.

E-mail:

office@khgorgas.com.ua
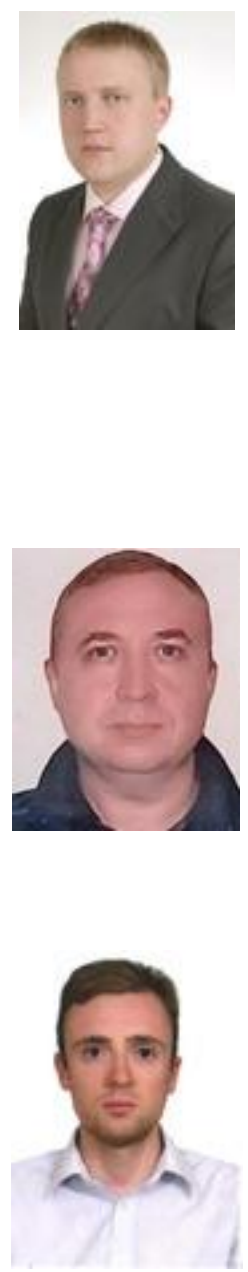

Редько

Андрей

\section{Александрович}

Доктор технических наук, профессор каф. ТГВ и ТВЕР, ХНУСА. Область научных интересов: использование возобновляемых источников энергии,

низкопотенциальной

энергии, тепломассообмен, термодинамика.

E-mail:

andrey.ua-mail@ukr.net

\section{Редько
Александрович}

Доктор технических наук, профессор каф. ТТДєМ, УГУЖТ. Область научных интересов: использование возобновляемых источников энергии, низкопотенциальной энергии, тепломассообмен, термодинамика.

E-mail:

germes_s2006@ukr.net

Пивненко Юрий
Александрович, к.т.н.,
ассистент каф. ТГВ и ТВЕР,
ХНУСА. Научные интересы:
низкопотенциальная энер-
гетика, кипящий слой, я,
теплофизика.
E-mail:
Pivnenko.Yura@gmail.com

[24] Knyshenko Y.V., Osadchiy A.E., Rashchetno-eksperemental'noe opredelenie efectivnosti vikhrevogo pyleulovitelya [Computational and experimental determination of the efficiency of a vortex dust collector]. Tekhnicheskaya mekhanika - Technical mechanics, 2012, no.4, pp. 5666. (In Russian).

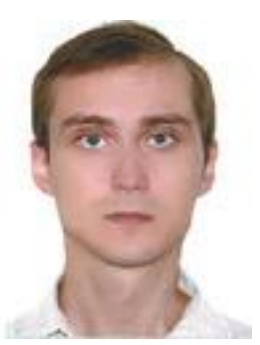

Бурда Юрий Александрович Кандидат технических наук, ассистент каф. ТГВ и ТВЕР, ХНУСА. Научные интересы: очистка вентиляционных выбросов, рациональное использование продуктов сгорания котлов, альтернативная энергетика, термодинамика.

E-mail:

burda.yurii.1991@gmail.com

\section{Гвоздецкий \\ Вадимович}

Кандидат технических наук, доцент каф. ТГВ и ТВЕР, ХНУСА. Область научных интересов: использование возобновляемых источников энергии, низкопотенциальной энергии, тепломассообмен, термодинамика.

E-mail: npp-tghv@ukr.net

Алфёров
Александрович
аспирант каф. ТГВ и ТВЕР,
ХНУСА. Научные интересы:
низкопотенциальная
энергетика, слоевое сжигание
твердого топлива, солнечная
энергия, теплофизика.
E-mail:
sergey.alf312@gmail.com

Алфёров

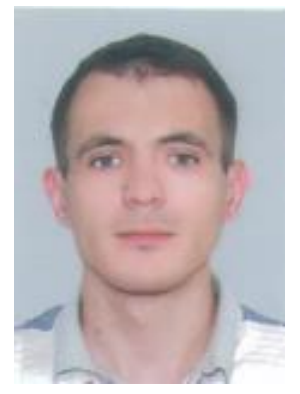

sergey.alf312@gmail.com 